\title{
Some Results on D-Homothetic Deformation On Almost Paracontact Metric Manifolds
}

\author{
Mehmet Solgun ${ }^{1}$ \\ ${ }^{1}$ Bilecik Seyh Edebali Universitesi
}

December 19, 2020

\begin{abstract}
In this work, we apply the notion of D-homothetic deformation on an almost paracontact metric manifolds and show that the structure after the deformation is also almost paracontact metric structure. Also, we state the classes of almost paracontact metric structures having parallel characteristic vector field and get some results about D- homothetic deformations on these classes.
\end{abstract}

\section{Hosted file}

wileyNJD-AMA.pdf available at https://authorea.com/users/383807/articles/499642-some-resultson-d-homothetic-deformation-on-almost-paracontact-metric-manifolds 